\title{
The Chemical Composition of $\alpha$ Cen A: Strong Lines and the ABO Theory of Collisional Line Broadening
}

\author{
Marianne T. Doyle ${ }^{\mathrm{A}, \mathrm{C}}$, Bernard J. O'Mara ${ }^{\mathrm{A} *}$, John E. Ross ${ }^{\mathrm{A}, \mathrm{C}}$, and Michael S. Bessell ${ }^{\mathrm{B}}$ \\ A Physical Department, University of Queensland, Brisbane QLD 4072, Australia \\ B Mount Stromlo Observatory, Australian National University, Weston Creek ACT 2611, Australia \\ C Corresponding authors. Email: mtdoyle@physics.uq.edu.au,ross@physics.uq.edu.au
}

Received 2003 October 17, accepted 2004 July 19

\begin{abstract}
The mean abundances of $\mathrm{Mg}, \mathrm{Si}, \mathrm{Ca}, \mathrm{Ti}, \mathrm{Cr}$, and $\mathrm{Fe}$ based on both strong and weak lines of $\alpha$ Cen $\mathrm{A}$ are determined by matching the observed line profiles with those synthesised from stellar atmospheric models and comparing these results with a similar analysis for the Sun. There is good agreement between the abundances from strong and weak lines.

Strong lines should generally be an excellent indicator of abundance and far easier to measure than the weak lines normally used. Until the development of the Anstee, Barklem, and O'Mara (ABO) theory for collisional line broadening, the uncertainty in the value of the damping constant prevented strong lines being used for abundance determinations other than in close differential analyses.

We found that $\alpha$ Cen A has a mean overabundance of $0.12 \pm 0.06$ dex compared to solar mean abundances. This result agrees remarkably well with previous studies that did not use strong lines or the ABO theory for collisional line broadening. Our result supports the conclusion that reliable abundances can be derived from strong lines provided this new theory for line broadening is used to calculate the van der Waals damping.
\end{abstract}

Keywords: stars: abundances — stars: individual $(\alpha$ Cen A $)$ - Sun: abundances

\section{Introduction}

The Alpha Centauri system has a special fascination for astrophysicists because it is the closest stellar system and its principle component, $\alpha$ Cen $\mathrm{A}$, has a spectral type very similar to the Sun, G2V. $\alpha$ Cen A is also one of the brightest stars in the sky, enabling spectra with a high spectral resolution and high signal to noise to be obtained. Most past analyses have concluded that the metal abundance of $\alpha$ Cen A is greater than that of the Sun. Analyses, such as Furenlid \& Meylan (1990) covering 26 elements from 500 lines and Neuforge-Verheecke \& Magain (1997) investigating 17 elements, concluded that the average metal overabundance compared to the Sun is $0.12 \pm(0.02-0.04)$ dex and 0.24 dex respectively. Another study (Chmielewski et al. 1992) concluded that $\alpha$ Cen A can be classed as a super metal rich star.

The importance of the present study is the use of the Anstee, Barklem, and O'Mara (ABO) theory (Barklem et al. 1998a) to determine van der Waals damping (VDW) for collisional line broadening for $\alpha$ Cen A. The ABO theory provides precise theoretical damping constants (as demonstrated in recent results for the analysis of strong lines in the solar spectrum by Allende Prieto et al. 2001), which enables the use of strong lines for which reliable laboratory $f$-values exist. Strong line wings are also relatively

\footnotetext{
${ }^{*}$ Deceased.
}

insensitive to the effects of turbulence in the atmosphere. Thus strong line wings, together with the ABO theory, may be used as reliable abundance indicators for elements where such strong lines exist.

The choice of lines for this project follows the investigation of Allende Prieto et al. (2001) for the Sun and includes weak neutral, weak ionised, and strong lines for six elements: $\mathrm{Mg}, \mathrm{Ca}, \mathrm{Si}, \mathrm{Ti}, \mathrm{Cr}$, and $\mathrm{Fe}$.

We employ high quality coude echelle CCD spectra observed using the 74-inch telescope at Mt Stromlo Observatory. The mass, distance, luminosity, and colours of $\alpha$ Cen $\mathrm{A}$ are used to determine its effective temperature, $T_{\text {eff }}$, and surface gravity, $\log \left(g_{\mathrm{s}}\right)$, necessary to customise existing atmospheric models to match $\alpha \mathrm{Cen} \mathrm{A}$. The model is based on Kurucz solar models (Kurucz 1979). The customised model, along with relevant atomic data, are used to synthesise line profiles for $\alpha$ Cen A. Abundances are determined by matching the observed profiles, following determination of turbulence parameters. Steps are taken to verify the $\alpha$ Cen A model used. A solar model based on the Holweger-Müller model (Holweger \& Müller 1974) is used to synthesise line profiles which are matched to the observational line profiles from the Jungfraujoch Atlas (Delbouille \& Roland 1963) to determine the solar mean abundance. The solar and $\alpha$ Cen A mean abundances are compared to find the mean under- or overabundance for $\alpha$ Cen A. 


\section{ABO Collisional Line Broadening Theory}

Collisional or VDW broadening is broadening resulting from the collision of atoms in the photosphere. It is especially important in cool stars such as our Sun and $\alpha$ Cen A that have predominately neutral hydrogen photospheres. This collisional broadening produces a Lorentzian line profile. The damping constant for collisional broadening is $\Gamma_{\text {coll }}$ and is included when synthesising the line profiles.

As discussed in Barklem et al. (1998a), until the 1970s formulations of VDW broadening developed by Lindholm (1942), Foley (1946), and Ünsold (1955) were used, and it was widely held that a better theory was needed. These theories deal with VDW interactions between perturbing hydrogen atoms and the absorbing atom. Although the term van der Waals broadening is still used today, it is really a misnomer as the actual line broadening theory is much more complex.

Brueckner (1971) introduced a perturbation theory formulation that involved long-range interaction where the electron exchange could be neglected but not the overlap in the atomic charge distribution, a point taken up in O'Mara (1976). O'Mara's work deals with collision broadening theory and draws from many sources to develop the beginning of what has come to be known as the ABO theory of line broadening. This theory has been further developed by Anstee, Barklem, and O'Mara (Anstee \& O'Mara 1991, 1995; Barklem \& O’Mara 1997; Barklem et al. 1998b). Further development of the ABO theory and its relevance to solar and late-type star abundances is ongoing with a code available on the world wide web (Barklem et al. 1998a) to calculate VDW.

\section{Observation and Data Reduction}

Our spectra were taken on three separate observational runs in 1996 June/July and 2001 May on the 74-inch telescope at Mt Stromlo Observatory. The 120 inch focal length coude camera was used with a 31.6 groove $\mathrm{mm}^{-1}$ echelle, cross-dispersed with a 150 lines $\mathrm{mm}^{-1}$ grating. Several different wavelength settings of the echelle grating and cross-disperser grating were used to obtain almost complete wavelength coverage from 4000 to $8000 \AA$.

The signal to noise ratio $(\mathrm{S} / \mathrm{N})$ varied with order across the CCD because of vignetting resulting from the crossdisperser not being near a pupil. The CCD has a gain of two electrons per ADU and most of the exposures were aimed at about 60000 electrons maximum. As the data are 60000-180000 electrons per resolution element, the nominal S/N ratio is 200-400. The actual resolution was 125000 estimated by measuring the width (FWHM) of the line at wavelength $8252.379 \AA$ from the thorium arc spectrum:

$$
R=\frac{\lambda}{\Delta \lambda}
$$

which corresponds to a velocity of $2.392 \mathrm{~km} \mathrm{~s}^{-1}$.

The reduction process included cleaning the raw spectra of cosmic rays and flat fielding. The spectral orders were extracted and any scattered light between the orders was removed. Extracted spectra from a nearby 'smooth spectrum' star, $\beta$ Cen, were divided through the spectra of the target star, $\alpha$ Cen A, to eliminate lines arising from the Earth's atmosphere. A thorium arc spectrum was used for wavelength calibration purposes. The spectra were smoothed to reduce noise and the continuum level was flattened. The wavelengths were also corrected for the radial velocity of $\alpha$ Cen A.

\section{The Atmospheric Models and Line Profiles}

Line profiles were synthesised for each line of interest and used to determine the abundances by fitting to the observed line profiles. Parameters such as macro- and microturbulence, VDW damping, energy levels of the transitions, $\log g_{f}$ values, and starting abundances were input to enable this direct matching process.

Two models and subsequent line profiles were used for $\alpha$ Cen A. Model AK is an interpolated Kurucz model (Kurucz 1979) grid; model AH is a scaled Holweger \& Müller solar model (Holweger \& Müller 1974). Both models use the calculated values for $\alpha$ Cen A's effective temperature and surface gravity. These two models are compared to verify the validity of using the interpolated Kurucz model in this project.

The third model, SH, is the Holweger \& Müller model (Holweger \& Müller 1974). This was used to synthesise line profiles to fit observed solar data obtained from the Jungfraujoch Atlas (Delbouville \& Roland 1963).

The initial models, based on published solar abundances, were used to compute line profiles to be compared with observations. If the line did not fit, the abundance of the element was adjusted and new number densities, opacities, and pressures were computed for a second iteration. This process was iterated to convergence yielding an abundance for each line. The temperature structure, $T_{\log \tau}$, of the initial atmosphere was not adjusted.

\subsection{Parameters and Calculations for the $\alpha$ Cen A Model Atmosphere and Synthesised Line Profiles}

\subsubsection{Effective Temperature and Surface Gravity}

Effective temperature, $T_{\text {eff }}$, and surface gravity, $\log \left(g_{\mathrm{s}}\right)$, are important parameters in the atmospheric model.

In Table 1 we list the measurements and derived data for $\alpha$ Cen A and the Sun that were used to determine the surface gravities and effective temperatures and were computed using the following equations:

$$
\begin{gathered}
\log g_{\mathrm{s}}=\log \left(\frac{G m}{r^{2}}\right) \\
T_{\text {eff }}=\left(\frac{L}{4 \pi r^{2} \sigma}\right)^{\frac{1}{4}}
\end{gathered}
$$

The derived values are in close agreement with those researched by other authors and are listed in Table 2. For $\alpha$ Cen A the resulting values are $T_{\text {eff }}=5784 \pm 5 \mathrm{~K}$ and $\log \left(g_{\mathrm{s}}\right)=4.28 \pm 0.01$. 
Table 1. Reported and calculated (marked with $*$ ) values used to customise the model atmosphere and produce synthesised line profiles for $\alpha$ Cen $A$ and the Sun

\begin{tabular}{|c|c|c|c|c|}
\hline Parameters & $\alpha \operatorname{Cen} A$ & Source & Sun & Source \\
\hline Angular diameter [arcsec] & $(86.2 \pm 2.3) \times 10^{-4 \mathrm{~A}}$ & Absolute IR photometry & & \\
\hline Parallax [arcsec] & $0.74212 \pm 0.0014^{\mathrm{B}}$ & Hipparcos Catalogue & & \\
\hline Apparent magnitude $V$ & $-0.01 \pm 0.006^{\mathrm{B}}$ & Hipparcos Catalogue & $-26.74 \pm 0.06^{\mathrm{C}}$ & See ref. \\
\hline$M_{v}$ & $4.35 \pm 0.006^{*}$ & $\operatorname{Scn} 4.1 .2$ & $4.83 \pm 0.002^{*}$ & $\operatorname{Sen} 4.1 .2$ \\
\hline Distance modulus & $-4.36 \pm 0.008^{*}$ & Scn 4.1.2 & $-31.57 \pm 0.06^{*}$ & $\operatorname{Sen} 4.1 .2$ \\
\hline Bolometric correction & $-0.07 \pm 0.01^{\mathrm{D}}$ & $\begin{array}{l}\text { Observational \& } \\
\text { synthetic } V \text {-band } \\
\text { spectra }\end{array}$ & $-0.07 \pm 0.01^{\mathrm{D}}$ & $\begin{array}{l}\text { Observational \& } \\
\text { synthetic } V \text {-band } \\
\text { spectra }\end{array}$ \\
\hline Bolometric magnitude & $4.2755 \pm 0.01^{*}$ & Calculated & $4.7620 \pm 0.01^{*}$ & $\operatorname{Sen} 4.1 .2$ \\
\hline Mass $[\mathrm{kg}]$ & $(2.1591 \pm 0.019) \times 10^{30 *}$ & Scn 4.1 .2 & $1.99 \times 10^{30 \mathrm{C}, \mathrm{F}}$ & See ref. \\
\hline $\operatorname{Mass}_{\odot}$ & $1.085 \pm 0.01^{\mathrm{E}}$ & From models & 1 & \\
\hline Radius [m] & $(8.6879 \pm 0.017) \times 10^{8 *}$ & $\operatorname{Scn} 4.1 .2$ & $(6.96 \pm 0.00026) \times 10^{8 \mathrm{C}}$ & See ref. \\
\hline $\operatorname{Radius}_{\odot}$ & $1.2483 \pm 0.0024^{*}$ & $\operatorname{Scn} 4.1 .2$ & 1 & \\
\hline Distance $[\mathrm{m}]$ & $4.1578 \pm 0.0078 \times 10^{16 *}$ & $\operatorname{Sen} 4.1 .2$ & $1.496 \times 10^{11 C, F}$ & See ref. \\
\hline Distance [pc] & $1.3456 \pm 0.0025^{*}$ & $\operatorname{Sen} 4.1 .2$ & $4.848 \times 10^{-6 * \mathrm{~F}}$ & $\operatorname{Sen} 4.1 .2$ \\
\hline Luminosity [W] & $6.0205 \pm 1 \times 10^{26 *}$ & $\operatorname{Scn} 4.1 .2$ & $3.846 \times 10^{26 \mathrm{C}, \mathrm{F}}$ & See ref. \\
\hline$L / L_{\odot}$ & $1.57 \pm 0.3^{*}$ & $\operatorname{Scn} 4.1 .2$ & 1 & \\
\hline Surface gravity $\left[\mathrm{m} \mathrm{s}^{-2}\right]$ & $(1.901 \pm 0.019) \times 10^{4 *}$ & $\operatorname{Scn} 4.1 .2$ & $(2.74 \pm 0.0002) \times 10^{4 *}$ & $\operatorname{Scn} 4.1 .2$ \\
\hline $\log g_{\mathrm{s}}$ & $4.28 \pm 0.01^{*}$ & $\operatorname{Scn} 4.1 .2$ & $4.44 \pm 0.00003^{*}$ & $\operatorname{Sen} 4.1 .2$ \\
\hline$T_{\text {eff }}[\mathrm{K}]$ & $5784.3 \pm 5.5^{*}$ & $\operatorname{Sen} 4.1 .2$ & $5778 \pm 1^{\mathrm{C}}$ & See ref. \\
\hline
\end{tabular}

A Blackwell \& Shallis (1977). ${ }^{B}$ ESA (1997). ${ }^{C}$ Ahrens (1995). ${ }^{D}$ Bessell et al. (1998). ${ }^{\text {E }}$ Demarque et al. (1986). ${ }^{\mathrm{F}}$ Error insignificant.

Table 2. Comparison of parameters for $\alpha$ Cen $A$

\begin{tabular}{|c|c|c|c|c|c|c|c|c|}
\hline Ref. & $T_{\text {eff }}[\mathrm{K}]$ & $\log \left(g_{\mathrm{s}}\right)$ & $\xi\left[\mathrm{km} \mathrm{s}^{-1}\right]$ & $M_{v}$ & $L / L_{\odot}$ & $r / r_{\odot}$ & $m / m_{\odot}$ & $\Delta \log A[\operatorname{dex}]$ \\
\hline This paper & $5784.3 \pm 5.5$ & $4.28 \pm 0.01$ & 1 & $4.35 \pm 0.006$ & $1.57 \pm 0.3$ & $1.25 \pm 0.0024$ & $1.085 \pm 0.01$ & $0.12 \pm 0.06$ \\
\hline Soderblom (1986) & $5770 \pm 20$ & & & & & 1.23 & & \\
\hline $\begin{array}{l}\text { Furenlid \& Meylan } \\
\text { (1990) }\end{array}$ & $5710 \pm 25$ & $4.0 \pm 0.2$ & $1.0 \pm 0.2$ & 4.38 & & 1.26 & 1.085 & $0.12 \pm 0.06$ \\
\hline Noels et al. (1991) & $5765 \pm 50$ & & & & 1.53 & & 1.085 & $0.25 \pm 0.02$ \\
\hline $\begin{array}{l}\text { Chmielewski et al. } \\
\text { (1992) }\end{array}$ & $5800 \pm 20$ & $4.31 \pm 0.02$ & 1 & $4.374 \pm 0.01$ & & & 1.085 & \\
\hline $\begin{array}{l}\text { Neuforge-Verheecke \& } \\
\text { Magain (1997) }\end{array}$ & $5830 \pm 30$ & $4.30 \pm 0.03$ & $1.09 \pm 0.11$ & & 1.53 & & $1.085 \pm 0.01$ & \\
\hline
\end{tabular}

\subsubsection{Input Data for Line Profile Synthesis}

The input data required for line synthesis are the energy levels, $\log \left(g_{f}\right)$, and VDW parameters taken from Allende Prieto et al. (2001, Table 3), and the starting solar abundances from Grevesse \& Sauval (1998, Table 4).

The parallax, $\pi$, and angular diameter, $\theta$, were used to calculate $\alpha$ Cen A's radius:

$$
\begin{gathered}
d=\frac{\left(d_{\odot}\right)}{\pi} \\
r=\frac{[d(\theta / 3600)(\pi / 180)]}{2}
\end{gathered}
$$

The apparent magnitude was used to calculate the effective temperature with the following steps:

$$
M_{v}=V-5 \log \frac{d}{10}
$$

$$
\begin{gathered}
M_{\mathrm{bol}}=M_{v}+B C \\
L=L_{\odot} 10^{\left[\left(M_{\mathrm{bol} \odot}-M_{\mathrm{bol}}\right) / 2.5\right]} \\
T_{\mathrm{eff}}=\left(\frac{L}{4 \pi r^{2} \sigma}\right)^{\frac{1}{4}}
\end{gathered}
$$

\subsection{Non-Thermal Broadening Parameters}

The non-thermal motions or turbulence that occur on a large scale compared with optical depth is macroturbulence, and on the small scale is microturbulence.

\subsubsection{Macroturbulence}

Macroturbulence will broaden the line profile but does not affect the line strength (equivalent width). The effects of stellar rotation (small) and instrumental profile are included as enhancements to the macroturbulence.

Several single lines and one blend of lines were chosen to determine the effective macroturbulence. Various values 
Table 3. Input parameters and abundances for $\alpha$ Cen A and the Sun

\begin{tabular}{|c|c|c|c|c|c|c|c|c|c|c|}
\hline \multirow[t]{2}{*}{$\lambda[\AA]$} & \multirow[t]{2}{*}{ Species $^{\mathrm{B}}$} & \multirow[t]{2}{*}{$E_{\text {low }}{ }^{\mathrm{B}}$} & \multirow[t]{2}{*}{$\log \left(g_{f}\right)^{\mathrm{B}}$} & \multicolumn{2}{|c|}{$\mathrm{VDW}^{\mathrm{B}}$} & \multicolumn{2}{|c|}{$\alpha \operatorname{Cen} A^{A}$} & \multicolumn{2}{|c|}{$\operatorname{Sun}^{\mathrm{A}}$} & \multirow[t]{2}{*}{$\Delta\langle A\rangle^{\mathrm{A}}[\operatorname{dex}]$} \\
\hline & & & & $\sigma$ & $\alpha$ & $W_{\lambda}[\mathrm{m} \AA]$ & A & $W_{\lambda}[\mathrm{m} \AA]$ & A & \\
\hline 4508.287 & FeII & 2.84 & -2.520 & 188 & 0.267 & 109.53 & 7.935 & 90.59 & 7.670 & 0.027 \\
\hline 4602.006 & FeI & 1.61 & -3.150 & 296 & 0.260 & 91.08 & 7.870 & 73.68 & 7.680 & 0.190 \\
\hline 4656.979 & FeII & 2.88 & -3.580 & 190 & 0.330 & 36.80 & 7.346 & 31.97 & 7.330 & 0.016 \\
\hline 4758.122 & TiI & 2.25 & 0.481 & 326 & 0.246 & 54.95 & 5.027 & 43.46 & 4.950 & 0.077 \\
\hline 4759.274 & TiI & 2.25 & 0.570 & 327 & 0.246 & 57.32 & 4.990 & 47.90 & 4.958 & 0.032 \\
\hline 4798.535 & TiII & 1.08 & -2.670 & 211 & 0.209 & 54.95 & 5.130 & 41.77 & 5.000 & 0.130 \\
\hline 4801.028 & CrI & 3.12 & -0.131 & 348 & 0.240 & 59.73 & 5.800 & 49.66 & 5.720 & 0.800 \\
\hline 4964.931 & $\mathrm{CrI}$ & 0.94 & -2.527 & 262 & 0.291 & 49.04 & 5.790 & 35.90 & 5.720 & 0.700 \\
\hline 5113.445 & TiI & 1.4 & -0.727 & 298 & 0.243 & 36.10 & 5.003 & 25.15 & 4.950 & 0.053 \\
\hline 5225.533 & $\mathrm{FeI}$ & 0.11 & -4.790 & 207 & 0.253 & 86.83 & 7.823 & 71.07 & 7.680 & 0.143 \\
\hline 5232.952 & FeI & 2.94 & -0.058 & 713 & 0.238 & 382.43 & 7.450 & 376.95 & 7.510 & -0.060 \\
\hline 5234.632 & FeII & 3.21 & -2.230 & 188 & 0.268 & 105.42 & 7.813 & 89.23 & 7.570 & 0.243 \\
\hline 5247.057 & FeI & 0.09 & -4.950 & 206 & 0.253 & 76.63 & 7.678 & 64.17 & 7.630 & 0.048 \\
\hline 5272.002 & $\mathrm{CrI}$ & 3.45 & -0.422 & 757 & 0.238 & 35.43 & 5.820 & 21.92 & 5.630 & 0.190 \\
\hline 5295.781 & TiI & 1.05 & -1.57 & 278 & 0.253 & 18.13 & 5.017 & 11.60 & 4.990 & 0.027 \\
\hline 5300.751 & CrI & 0.98 & -2.129 & 329 & 0.263 & 71.50 & 5.890 & 54.71 & 5.720 & 0.170 \\
\hline 5312.859 & CrI & 3.45 & -0.562 & 751 & 0.238 & 27.21 & 5.776 & 18.75 & 5.680 & 0.96 \\
\hline 5336.793 & TiII & 1.58 & -1.630 & 272 & 0.314 & 90.84 & 5.300 & 73.21 & 5.050 & 0.250 \\
\hline 5418.773 & TiII & 1.58 & -2.110 & 270 & 0.315 & 61.42 & 5.140 & 47.55 & 4.980 & 0.160 \\
\hline 5490.154 & TiI & 1.46 & -0.877 & 374 & 0.262 & 28.78 & 4.984 & 19.64 & 4.945 & 0.39 \\
\hline 5665.557 & SiI & 4.92 & -1.940 & 1772 & 0.222 & 56.34 & 7.680 & 39.12 & 7.480 & 0.200 \\
\hline 5684.490 & SiI & 4.95 & -1.550 & 1798 & 0.221 & 77.74 & 7.610 & 66.87 & 7.500 & 0.110 \\
\hline 5690.425 & SiI & 4.93 & -1.770 & 1772 & 0.222 & 62.79 & 7.616 & 52.84 & 7.520 & 0.096 \\
\hline 5701.106 & SiI & 4.93 & -1.950 & 1768 & 0.222 & 55.19 & 7.680 & 39.91 & 7.510 & 0.170 \\
\hline 5708.402 & SiI & 4.95 & -1.370 & 1787 & 0.222 & 95.29 & 7.640 & 84.96 & 7.537 & 0.103 \\
\hline 5787.922 & CrI & 3.32 & -0.083 & 1097 & 0.291 & 87.12 & 6.203 & 48.17 & 5.660 & 0.543 \\
\hline 5866.457 & TiI & 1.07 & -0.784 & 259 & 0.262 & 59.83 & 5.120 & 43.38 & 4.980 & 0.140 \\
\hline 5916.254 & $\mathrm{FeI}$ & 2.45 & -2.990 & 341 & 0.238 & 64.02 & 7.710 & 52.80 & 7.650 & 0.060 \\
\hline 5922.115 & TiI & 1.05 & -1.410 & 313 & 0.242 & 28.10 & 5.065 & 17.82 & 5.000 & 0.065 \\
\hline 5948.545 & SiI & 5.08 & -1.130 & 1875 & 0.222 & 107.51 & 7.663 & 94.74 & 7.506 & 0.157 \\
\hline 6082.715 & FeI & 2.22 & -3.570 & 306 & 0.271 & 47.38 & 7.697 & 33.24 & 7.590 & 0.107 \\
\hline 6092.799 & TiI & 1.89 & -1.323 & 398 & 0.239 & 7.92 & 5.125 & 3.77 & 4.96 & 0.165 \\
\hline 6151.623 & FeI & 2.18 & -3.300 & 277 & 0.263 & 62.73 & 7.711 & 49.26 & 7.61 & 0.101 \\
\hline 6161.297 & CaI & 2.52 & -1.266 & 978 & 0.257 & 79.49 & 6.540 & 66.02 & 6.409 & 0.131 \\
\hline 6162.183 & CaI & 1.89 & -0.097 & 878 & 0.236 & 280.96 & 6.350 & 277.82 & 6.350 & 0 \\
\hline 6166.441 & CaI & 2.52 & -1.142 & 976 & 0.257 & 81.87 & 6.454 & 75.14 & 6.420 & 0.034 \\
\hline 6173.342 & FeI & 2.2 & -2.880 & 281 & 0.266 & 81.34 & 7.740 & 70.01 & 7.664 & 0.076 \\
\hline 6200.321 & FeI & 2.61 & -2.440 & 350 & 0.235 & 85.81 & 7.750 & 74.75 & 7.680 & 0.070 \\
\hline 6258.109 & TiI & 1.44 & -0.299 & 355 & 0.237 & 62.19 & 5.010 & 51.87 & 4.980 & 0.030 \\
\hline 6297.801 & FeI & 2.2 & -2.750 & 278 & 0.264 & 86.68 & 7.700 & 76.60 & 7.650 & 0.050 \\
\hline 6371.361 & SiII & 8.12 & -0.000 & 389 & 0.189 & 43.34 & 7.850 & 35.22 & 7.520 & 0.330 \\
\hline 6432.684 & FeII & 2.89 & -2.510 & 174 & 0.270 & 53.76 & 7.600 & 43.42 & 7.449 & 0.151 \\
\hline 6455.604 & CaI & 2.52 & -1.290 & 365 & 0.241 & 69.90 & 6.490 & 56.85 & 6.350 & 0.140 \\
\hline 6481.878 & FeI & 2.28 & -2.980 & 308 & 0.243 & 76.78 & 7.757 & 65.20 & 7.680 & 0.077 \\
\hline 6498.945 & FeI & 0.96 & -4.700 & 226 & 0.253 & 57.82 & 7.740 & 43.20 & 7.658 & 0.082 \\
\hline 6499.656 & CaI & 2.52 & -0.818 & 364 & 0.239 & 101.06 & 6.600 & 92.17 & 6.500 & 0.100 \\
\hline 6516.086 & FeII & 2.89 & -3.380 & 174 & 0.270 & 45.07 & 7.270 & 53.99 & 7.550 & -0.280 \\
\hline 6750.161 & FeI & 2.42 & -2.620 & 335 & 0.241 & 111.89 & 8.150 & 79.07 & 7.695 & 0.455 \\
\hline 6978.861 & $\mathrm{FeI}$ & 2.48 & -2.500 & 337 & 0.241 & 108.08 & 8.000 & 82.04 & 7.662 & 0.338 \\
\hline 7357.735 & TiI & 1.44 & -1.06 & 329 & 0.244 & 29.57 & 5.060 & 20.77 & 5.030 & 0.030 \\
\hline 7515.836 & FeII & 3.9 & -3.450 & 187 & 0.271 & 23.33 & 7.750 & 14.73 & 7.517 & 0.233 \\
\hline 7680.271 & SiI & 5.86 & -0.590 & 2107 & 0.495 & 101.18 & 7.640 & 101.78 & 7.610 & 0.030 \\
\hline 7711.730 & FeII & 3.9 & -2.450 & 186 & 0.264 & 58.42 & 7.560 & 50.79 & 7.430 & 0.130 \\
\hline 7918.387 & SiI & 5.95 & -0.510 & 2934 & 0.232 & 108.85 & 7.600 & 102.00 & 7.504 & 0.096 \\
\hline 8327.067 & $\mathrm{FeI}$ & 2.20 & -1.525 & 258 & 0.247 & 226.21 & 7.660 & 198.38 & 7.621 & 0.039 \\
\hline 8542.120 & CaII & 1.7 & -0.463 & 291 & 0.275 & 3206.68 & 6.360 & 3274.47 & 6.360 & 0 \\
\hline 8662.169 & CaII & 1.69 & -0.723 & 291 & 0.275 & 2568.95 & 6.420 & 2566.40 & 6.380 & 0.040 \\
\hline 8806.778 & MgI & 4.33 & -0.120 & 531 & 0.292 & 679.65 & 7.900 & 562.10 & 7.680 & 0.220 \\
\hline
\end{tabular}

\footnotetext{
${ }^{\text {A }}$ This paper. ${ }^{\mathrm{B}}$ Allende Prieto et al. (2001).
} 
Table 4. Mean abundances and mean overabundances for $\alpha$ Cen A models (AK) and Sun model (SH)

\begin{tabular}{lcccc}
\hline Element & Grevesse \& Sauval (1998) & \multicolumn{1}{c}{$\alpha \operatorname{Cen~A-\langle A\rangle }$} & \multicolumn{1}{c}{ Solar $-\langle A\rangle$} & $\Delta\langle A\rangle[$ dex] \\
\hline $\mathrm{Mg}$ & $7.58 \pm 0.05$ & $7.9(1$ line $)$ & 7.6800 & 0.200 \\
$\mathrm{Ca}$ & $6.36 \pm 0.02$ & $6.459 \pm 0.092$ & $6.396 \pm 0.054$ & $0.064 \pm 0.017$ \\
$\mathrm{Si}$ & $7.55 \pm 0.05$ & $7.664 \pm 0.0756$ & $7.521 \pm 0.0370$ & $0.144 \pm 0.011$ \\
$\mathrm{Ti}$ & $5.02 \pm 0.06$ & $5.075 \pm 0.088$ & $4.983 \pm 0.032$ & $0.092 \pm 0.019$ \\
$\mathrm{Cr}$ & $5.67 \pm 0.03$ & $5.880 \pm 0.163$ & $5.688 \pm 0.038$ & $0.192 \pm 0.029$ \\
$\mathrm{Fe}$ & $7.50 \pm 0.05$ & $7.714 \pm 0.197$ & $7.599 \pm 0.098$ & $0.115 \pm 0.029$ \\
\multicolumn{4}{c}{$\alpha$} \\
\hline
\end{tabular}

were tested on each single line to see if a common value can be used to match the general shape of the observed line. When the value for the effective macroturbulence was determined, that value was used on the blended lines with no effect on the equivalent width of the synthesised line profile.

The $\alpha$ Cen A value for the effective macroturbulence was determined to be $3.3 \mathrm{~km} \mathrm{~s}^{-1}$. This includes $2.4 \mathrm{~km} \mathrm{~s}^{-1}$ due to instrumental effects and an atmospheric macroturbulence of $2.3 \mathrm{~km} \mathrm{~s}^{-1}$. This indicates that most of the observed line broadening effects near the line core are from the large scale motions in the photosphere. Natural, Stark, and rotational broadening are insignificant for the lines chosen for this study.

The effective macroturbulence to match the line profiles from the AH solar model to the observed solar data is $1.6 \mathrm{~km} \mathrm{~s}^{-1}$

\subsubsection{Microturbulence}

Values for the microturbulence were determined by using weak neutral, weak ionised, and strong Fe lines. The values fitted are $1.08 \pm 0.2 \mathrm{~km} \mathrm{~s}^{-1}$ for $\alpha$ Cen A with the AK model and $0.85 \mathrm{~km} \mathrm{~s}^{-1}$ for the Sun with the SH model.

\subsection{Validity of the Model}

To judge whether the interpolated model AK is valid, comparison with an empirical model based on observations is useful.

The equivalent widths and profiles from model $\mathrm{AK}$ were compared to those produced with model $\mathrm{AH}$, a scaled solar empirical Holweger \& Müller model (Holweger \& Müller 1974). Eight lines were chosen and a good match of line profiles and equivalent widths was obtained.

Another method for comparing the validity of model AK is to plot $T_{\log \tau}$ versus $\log \tau_{\mathrm{o}}$ for both models. As can be seen from Figure 1 there is close agreement for $\log \tau_{\mathrm{o}}$ in the range -3 to +0.6 . This is the range of optical depths that is covered in this research. A direct comparison can be seen in Figure 2 where a strong line, CrI at $4801 \AA$, for both models has been overlaid and matches exactly.

\section{Analysis}

\subsection{Determining the Mean Abundance}

Fitting the shape of the synthesised line profiles from model AK against that of the observations is straightforward for most of the lines.

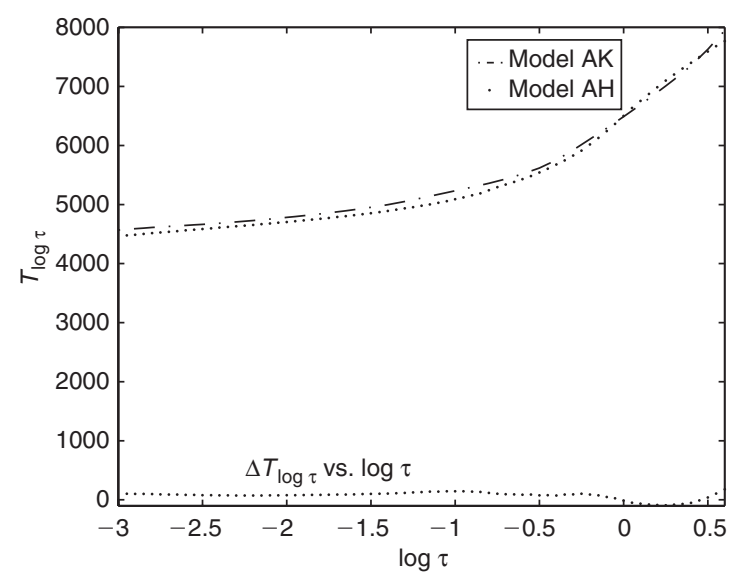

Figure 1 Temperature versus optical depth: comparison of the AK and $\mathrm{AH}$ models to test for validity.

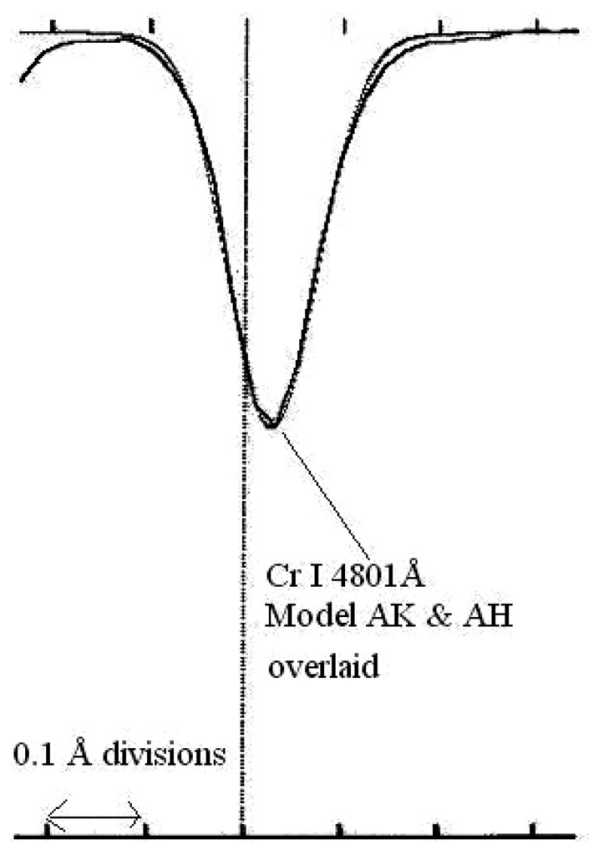

Figure 2 Model AK \& AH line profiles overlaid for CrI 4801 Å.

For all lines, care was taken to ensure that the areas of the synthesised and observed line profiles were equivalent, allowing for differences in the exact matching of the line profile's core and wings. For two very weak lines and all the strong lines, extra care was needed. 

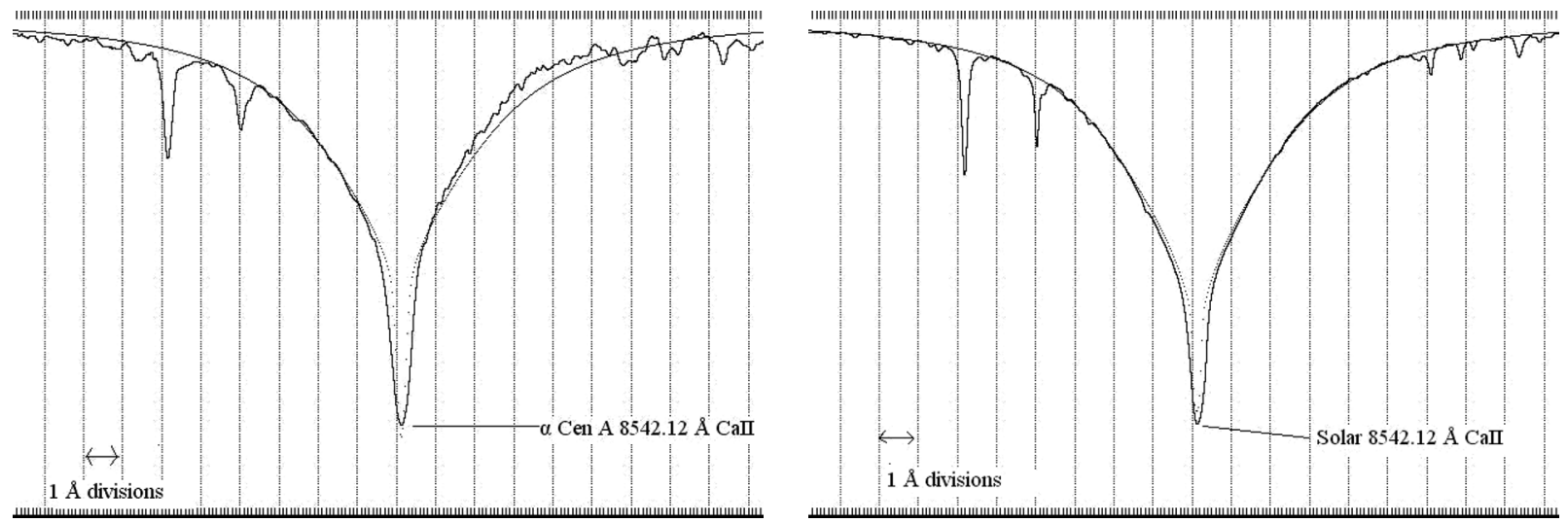

Figure 3 Fitting model line profile to observed data for $\alpha$ Cen A and the Sun to determine equivalent width and abundance for strong CaII line $8542.120 \AA$ (scale: 1 Å divisions).

In the case of the strong lines, the core and wings could not be matched simultaneously. In this case, the wings were matched, as per the ABO theory, and the core of the model's line profile was extended as low as possible. Care was taken that this technique was followed in both models' line profiles for those lines affected.

As can be seen in Figure 3 the right hand wing of the line profile for the observed data for $\alpha$ Cen $\mathrm{A}$ is higher than that of the synthesised line profile. This does not happen in the Sun's line profile. The problem then is with the reduction process. Care was taken to ensure that features like this were compensated for in the determination of the abundances.

\subsection{Results}

The mean abundances were determined from $1 \mathrm{Mg}, 7 \mathrm{Ca}$, $9 \mathrm{Si}, 13 \mathrm{Ti}, 6 \mathrm{Cr}$, and $22 \mathrm{Fe}$ lines. Each element is represented by species that cover weak neutral, weak ionised, and strong lines, except for $\mathrm{Mg}$ which is represented by one strong line. $\alpha$ Cen A shows a mean overabundance of $0.12 \pm 0.06 \mathrm{dex}$. The error was calculated from the variations in the individual species abundances.

These abundances are larger than those used for starting abundances from Grevesse \& Sauval (1998). The individual equivalent widths $W_{\lambda}$, abundance $A$, and $\Delta$ abundances $\Delta A$ for $\alpha$ Cen $\mathrm{A}$ and the Sun are listed in Table 3. The mean abundance $\langle A\rangle$, the $\Delta$ mean abundances $\Delta\langle A\rangle$, along with the starting abundances for $\alpha$ Cen A and the Sun for each element and the final mean overabundance, are listed in Table 4.

\section{Summary and Discussions}

The mean abundance for the six elements investigated in the chemical composition of $\alpha$ Cen $\mathrm{A}$ are $\mathrm{Mg}=7.9^{1}$, $\mathrm{Ca}=6.46 \pm 0.09, \quad \mathrm{Si}=7.66 \pm 0.07, \quad \mathrm{Ti}=5.07 \pm 0.09$, $\mathrm{Cr}=5.88 \pm 0.16$, and $\mathrm{Fe}=7.71 \pm 0.20 \mathrm{dex}$. This leads to a mean abundance of $0.12 \pm 0.06 \mathrm{dex}$ with respect to the Sun.

\footnotetext{
${ }^{1}$ Only one line was analysed, hence there is no error value.
}

Previous studies were not able to use strong lines as no reliable theory existed to calculate the collisional broadening of these lines. For this project the development of the ABO theory (Barklem et al. 1998a) to calculate the VDW damping enabled us to use strong lines in determining the mean abundances of $\alpha$ Cen A compared to the Sun.

The $\alpha$ Cen A parameters used are $T_{\text {eff }}=5784.3 \pm 5.5 \mathrm{~K}$ and $\log \left(g_{\mathrm{s}}\right)=4.28 \pm 0.01$. Two models for $\alpha$ Cen A were used, with the second one ( $\mathrm{AH})$ a scaled solar model for comparison with the first (AK) to verify the validity of using an interpolated Kurucz model (Kurucz 1979). Once the use of model AK was validated, this model was used to synthesise line profiles for $\alpha$ Cen A to match the observed line profiles.

Solar abundances were determined from comparing observed line profiles from the Jungfraujoch Atlas with those of the Holweger-Müller solar model (Holweger \& Müller 1974).

The result of this study, that $\alpha$ Cen A is overabundant with respect to the Sun and can be included with other metal rich stars, agree with those of previous studies (Noels et al. 1991; Chmielewski et al. 1992; Neuforge 1993; Neuforge-Verheecke \& Magain 1997) with an exact agreement of mean overabundance of $0.12 \pm 0.06$ dex by Furenlid \& Meylan (1990). This mean overabundance indicates that $\alpha$ Cen A did not originate in the same cloud as the Sun but from material that is more enriched by stellar nuclear processing.

Previous studies did not use strong lines or the ABO theory. Our results support the determination that reliable abundances can be derived from strong lines provided that the $\mathrm{ABO}$ theory is used to calculate the VDW damping.

By using the ABO theory for strong lines, the analysis of spectra, construction of model atmospheres and the subsequent synthesised line profiles, and the chemical composition of apparently faint stars, such as those in external galaxies, can be determined. All galaxies contain cool F-, G-, and K-type stars whose spectra contain strong metallic lines. Previously these lines were not able to be used for absolute abundances due uncertainties in the theory for calculating the VDW damping. With the ABO theory now firmly established and reliable model 
atmospheres existing for cool stars, it will be possible to extend reliable abundance analyses to more and more distant galaxies using strong lines.

\section{Acknowledgments}

M.T.D. would like to acknowledge and thank Paul Barklem for the use of the VDW damping values calculated from the $\mathrm{ABO}$ theory and the Astrophysics Group at the University of Queensland. The anonymous referees are thanked for their constructive comments.

\section{References}

Ahrens, T. J. (ed.) 1995, Global Earth Physics: A Handbook of Physical Constants (Washington DC: American Geophysical Union)

Allende Prieto, C., Barklem, P. S., Asplund, M., \& Ruiz Cobo, B. 2001, ApJ, 558, 830

Anstee, S. D., \& O'Mara, B. J. 1991, MNRAS, 253, 549

Anstee, S. D., \& O'Mara, B. J. 1995, MNRAS, 276, 859

Barklem, P. S., Anstee, S. D., \& O’Mara, B. J. 1998a, PASA, 15, 336

Barklem, P. S., \& O'Mara, B. J. 1997, MNRAS, 290, 102

Barklem, P. S., O’Mara, B. J., \& Ross, J. E. 1998b, MNRAS, 296, 1057
Bessell, M. S., Castelli, F., \& Plez, B. 1998, A\&A, 333, 231 Blackwell, D. E., \& Shallis, M. J. 1977, MNRAS, 180, 177

Brueckner, K. A. 1971, ApJ, 169, 621

Chmielewski, Y., Friel, E., Cayrel de Strobel, G., \& Bentolila, C. 1992, A\&A, 263, 219

Delbouille, L., \& Roland, C. 1963, Atlas Photometrique du Spectre Solaire de $\lambda 7498$ à $\lambda 12016$ (Liège)

Demarque, P., Guenther, D. B., \& van Altena, W. F. 1986, ApJ, 300,773

ESA 1997, The Hipparcos and Tycho Catalogues, ESA SP-1200

Foley, H. M. 1946, PhRv, 69, 621

Furenlid, I., \& Meylan, T. 1990, ApJ, 350, 827

Grevesse, N., \& Sauval, A. J. 1998, SSRv, 85, 161

Holweger, H., \& Müller, E. A. 1974, SoPh, 39, 19

Kurucz, R. L. 1979, ApJS, 40, 1

Lindholm, E. 1942, PhD Thesis, Uppsala University

Neuforge, C. 1993, A\&A, 268, 650

Neuforge-Verheecke, C., \& Magain, P. 1997, A\&A, 328, 261

Noels, A., Grevesse, N., Magain, P., Neuforge, C., Baglin, A., \& Lebreton, Y. 1991, A\&A, 247, 91

O'Mara, B. J. 1976, MNRAS, 177, 551

Ünsold, A. 1955, Physik der Stern Atmosphären (2nd ed.) (Berlin: Springer)

Soderblom, D. R. 1986, A\&A, 158, 273 